\title{
Neurologic Music Therapy in Stroke Rehabilitation
}

\author{
Michael H. Thaut • Gerald C. McIntosh
}

Published online: 2 April 2014

(C) Springer Science + Business Media New York 2014

\begin{abstract}
Based on insights from brain research in music, neurologic music therapy (NMT) has been established as a new model for music in therapy and medicine. Standardized clinical interventions are based on clusters of research evidence and established learning principles in motor, speech/language, and cognitive training. The research support for NMT in stroke rehabilitation has been growing rapidly over the past 20 years. This paper will review research data and clinical applications for neurorehabilitation in the speech/language, cognitive and sensorimotor domains.
\end{abstract}

Keywords Neurologic music therapy $\cdot$ Stroke ·

Rehabilitation $\cdot$ Neuroscience $\cdot$ Music $\cdot$ Rhythm

\section{Introduction}

Over the past two decades, researchers have begun to elucidate the neural substrates of musical functions in the human brain. In our own research, rhythm perception and production have been a primary focus of these efforts, because they form the most important structural element in the language of music. The study of rhythm has produced insights into musical time processing and temporal information processing in the human brain. These investigations have led to a fundamental need to rethink the role of music

M. H. Thaut $(\square)$

Center for Biomedical Research in Music, Colorado State

University, Fort Collins, CO, USA

e-mail: Michael.Thaut@ColoState.EDU

G. C. McIntosh

Department of Neurology, University of Colorado Health, Fort Collins, CO, USA in therapy and medicine, tapping the capacity of music to serve as a powerful complex auditory stimulus capable of engaging the brain in retraining neural and behavioral functions that can then be applied to nonmusical functional contexts in therapy and medicine.

Traditionally, music in therapy has been based mostly on social science models in which the cultural role of music is interpreted as an effective facilitator for therapeutic concepts of "well-being, emotional response, and relationship building." Neuroscience research in music has created new insights into the therapeutic benefits of music, shifting the models of music in therapy from social science and interpretative models to neuroscience and perceptual models. That is, they are based on how the structures and patterns in music engage the brain in ways that can be meaningfully translated and generalized to nonmusical therapeutic learning and training. In other words, the brain that engages in music is changed by engaging in music. This reciprocal relationship of music and brain functiondiscovered in the mid-1990s within the larger context of a very fascinating line of research demonstrating the experience-dependent plasticity of the brain-is one of the most powerful motors of change in the historical paradigm of music therapy. These new findings suggest that music can stimulate complex cognitive, affective, and sensorimotor processes in the brain that can then be generalized and transferred to nonmusical therapeutic applications.

The clinical answer to these conceptual changes has been the development of neurologic music therapy (NMT). Unlike the traditional socio-cultural model of music therapy, NMT uses the perception of auditory structures and patterns in music as cues to retrain brain function. NMT requires advanced certification training and consists of standardized treatment techniques which are based on clusters of research evidence in the areas of motor, speech/ 
language, and cognitive rehabilitation. In the following we will review research underlying NMT techniques specifically addressing motor and speech and language recovery in stroke rehabilitation.

\section{Studies in Gait Rehabilitation}

The first field of inquiry in which breakthrough data emerged for a new understanding of the role and function of music in therapy came in motor-control research. Several studies conducted since the mid-1990s demonstrate impressively that rhythmic entrainment of motor function can actively facilitate the recovery of movement in patients with stroke. Early landmark studies by Paltsev and Elner [1] and Rossignol and Melvill Jones [2] had shown evidence for the complex physiological interactions between the auditory and the motor system. Most studies that appeared in the 1970s and 1980s had reported beneficial effects of rhythm and music on motor development and movement performance using behavioral and motivational measures. Sensorimotor research focusing on motor learning principles or physiological mechanisms had focused almost exclusively on the role of visual and proprioceptive modalities in the control of movement.

It took the studies conducted by Paltsev and Elner [1] and Rossingol and Melvill Jones [2] to show evidence for the existence of auditory-motor pathways (e.g., via reticulospinal connections) that could influence threshold excitability of motor neurons, creating a readiness or priming effect on the segmental motor system via auditory input. Rossignol and Melvill Jones also demonstrated that this priming effect could turn into a functionally facilitating timing effect of muscle activation patterns during auditory rhythmic cuing of rhythmic leg movement.

The first study investigating the influence of auditory rhythm on gait was carried out by our group on healthy subjects. The data showed a strong synchronization effect between the timing of the beat cues and the leg movements. Furthermore, from a physiological point, significant reductions in electromyographic variability of the leg muscles (gastrocnemius) became evident in rhythmically cued gait of healthy subjects [3]. These data set the stage for a follow-up study with hemiparetic ischemic stroke patients 6-18 months post stroke [4]. Results showed significant entrainment results using auditory rhythm as a pacemaker. Basic gait parameters such as velocity, step cadence, and swing symmetry all improved with rhythmic cuing. In addition, EMG variability of the gastrocnemius muscle was reduced significantly in the paretic leg, showing more economical motor unit recruitment patterns.
For a better understanding of what mechanisms might be driving the almost instantaneous entrainment effect and associated improvements in functional gait patterns, we carried out kinematic research based on digital video motion analysis [5]. Results showed significant gains in stride length and symmetry as well as reductions in lateral center of mass (COM) displacement and increases in vertical COM, indicating gait patterns closer to the norms. One of the important outcomes of this analysis was the change not just in temporal gait measures but also in spatial kinematics such COM displacements. Using the entrainment paradigm as the basis for a six-week gait therapy investigation, we investigated long-term training effects with rhythmic auditory stimulation (RAS) in patient samples entering the study approximately two weeks post stroke. The study samples consisted of patients with right and left hemispheric ischemic and hemorrhagic strokes in the middle cerebral artery and internal capsule distribution. Velocity, stride length, and stride symmetry were significantly higher than for conventional NDT/Bobath based gait therapy [6]. Besides improvements in kinematic gait parameters, a central physiological effect of auditory rhythm on EMG patterns was found in reduction of amplitude variability of the gastrocnemius muscle. In a larger international multiple center study (US/Germany) replicating the study in 1997 with a shorter intervention period of three weeks-similar results were confirmed with the exception that the magnitude of the changes in the study groups were proportionally about $30 \%$ smaller compared to six weeks [7••].

RAS has been researched by other research groups. Similar data were found for hemiparetic stroke patients by Schauer and Mauritz [8] and Schauer et al. [9] using RAS during treadmill walking. A study by Ford et al. [10] found significant improvements for gait and associated arm swing in patients with stroke. Abiru et al. [11] found significant improvements for RAS over conventional gait training by physical therapy in stride and step length, cadence, and velocity. Improvements in the symmetry index in patients with thalamic lesions were not consistent indicating the lesion site needs to be taken into consideration. Several research studies by Roerdink et al. [12-14•] confirmed and extended our previous RAS findings in gait rehabilitation for hemiparetic stroke.

Taken together, the physiological data suggest that the periodicity of the pulsed rhythmic auditory cue had a modulatory effect on motor neuron activity entraining neural activation patterns into more regular and more synchronized patterns, resulting in more consistent timing and motor unit recruitment. Because these EMG enhancements were always linked with significant kinematic improvements, one can postulate that the rhythmic cues were acting on centrally mediated mechanisms of 
motor control, possibly via resonance coupling of motor neuron activation patterns to the physiological coding of rhythm in the auditory pathway. Furthermore, rhythmic time cues provide an anticipatory time reference for onset, duration, and completion of movement. In several psychophysical studies, we were able to show that rhythmic motor entrainment is not based on entrainment to the beat event but on period entrainment, i.e., the rhythmic time interval provides a continuous time reference for the motor system to map and scale the movement duration unto the duration of the rhythmic cue. In the period entrainment model, the movement endpoints can have some slack time around the actual synchronization to the beat, allowing for adaptive flexibility in movement control $[15,16]$.

\section{Studies in Arm Rehabilitation}

In order to extend rhythmic entrainment paradigms from gait research to therapy for other motor functions, two NMT techniques turn functional movements, e.g., in the upper extremities or in full body coordination, into cyclical repetitive movement patterns that can be cued by auditory rhythm. Patterned Sensory Enhancement (PSE) regulates functional movement by translating the temporal, spatial, and force-dynamic components of movement kinematics into sound patterns, similar to sonification patterns in high performance athletic training (Schaffert et al.), and then plays them back to provide feedback and feedforward regulation for enhanced motor control. The second technique, Therapeutic Instrumental Playing (TIMP), maps functional movements unto musical instruments (mostly percussion, keyboard, or digital sound surfaces) and engages the patient in practicing motions repetitively and cyclically via musical instruments. Patient playing is often cued by an external rhythmic cue for anticipatory time control. TIMP offers three advantages over nonmusical motor training:

- Priming of the motor system due to the enriched sound environment

- Auditory feedback for spatial movement control (knowledge of results from sound when the instrument has been struck correctly)

- Anticipation and timing structure for movement from the external rhythmic cue and from integrating the external cue (feedforward) with the patient produced sound patterns (feedback)

Several studies have researched aspects of TIMP and PSE successfully in hemiparetic arm rehabilitation for CVA [PSE: 17-19•] [TIMP: 20-26•, 27, 28••]. Whitall et al. [20], Thaut et al. [17], and Luft et al. [21] demonstrated increases in ranges of motion with increases in isometric arm-joint strength and significant improvements on several standardized tests of arm function. Also, decreases in variability and increases in speed of movement times were found, as well as-most noteworthysmoothing of trajectory variability during reaching movements. By studying the underlying velocity and acceleration profiles during movements, a very important insight into understanding the effect of auditory rhythm on motor control was developed. There had been very strong evidence that non-temporal movement parameters, such as stride length in gait or movement trajectories of upper and lower limb joints (e.g., wrist, knee), improved during rhythmic cuing. One basic conclusion was that enhanced time stability across the duration of the movement during rhythmic cuing - by way of rhythm providing a continuous time reference based on its period information-also enhanced spatial-positional control of movement. However, a conceptual link to connect temporal cuing to spatiodynamic parameters of motor control was missing. Analyses of the acceleration and velocity profiles of joint motions during rhythmic cuing offered an intriguing explanation by linking the different parameters of movement control into an interdependent system that could be accessed and modulated by time.

The consistent evidence for smoothing of velocity and acceleration profiles of joint motions during rhythmic cuing suggests that rhythm enhances the control of velocity and acceleration by scaling movement time. Velocity and acceleration, however, are mathematical time derivatives of the spatial parameter of position. Thus, in working our theory backward, we reasoned that by fixating time through a rhythmic interval, for a movement from point $A$ to point B the subject's internal timekeeper now had a precise reference interval, with time information present at any stage or moment of the movement. This time information allows the brain to map and scale smoother parameters of position change (i.e., velocity and acceleration) across the entire movement interval (e.g., reaching target points in space for arm movements, etc.). Changes in velocity and acceleration profiles, however, must be reflected in the position-time curves of the movement. This can be described mathematically as an optimization problem. If we assume that the brain uses some optimization strategy to control movement, it is possible to show, in certain cases, that such optimization implies scaling of the resulting movement over time. The immediate consequence of this assertion is that matching the period of a cyclical movement to the period of an external timekeeper will result in the regulation of the entire movement trajectory. Once the time constraint has been added, the brain is presented with a well-defined optimization problem: how to move from point $\mathrm{A}$ to point $\mathrm{B}$ in a fixed time interval 
while maximizing precision and minimizing some objective cost function for the body associated with making the movement.

\section{Studies in Speech and Language Rehabilitation}

From a biomedical perspective, two shared functions of music, speech, and language are important: (1) the aural and production features shared by spoken language and musical vocalization in singing and (2) the ability of both "systems" to embed communicative functions in the auditory modality. One of the earliest systematic applications of music to the recovery of speech function was developed in the early 1970 s by using singing with patients who suffered from expressive (Broca's) aphasia. The term "melodic intonation therapy" (MIT) was coined to describe this technique [29-31]. MIT is the most frequently used speech/language therapy technique used in stroke rehabilitation.

There is a significant amount of research starting in the mid-1970s showing the efficacy of MIT with non-fluent expressive aphasia [32-47]. However, as a word of caution, most of these research studies have been conducted with small sample sizes due to the fact that it is not easy to find homogenous study samples in aphasia research in terms of lesion site and symptom consistency. Experiments by Stewart et al. [48] used transcranial magnetic stimulation to disrupt speech and singing. Left hemispheric stimulation disrupted speech, but right hemispheric stimulation did not disrupt singing. These findings raise questions about the neural circuitry for singing, which may be more diffusely located and, as such, harder to disrupt than the highly localized speech centers in the left hemisphere. Several studies, e.g., Belin et al. [32], Schlaug et al. [41•] and Breier et al. [35•] have shown evidence for neuroplasticity induced by MIT, rerouting speech pathways from the damaged left to the language-capable regions of the right hemisphere. Long-term MIT training may also show the reverse, re-activating left hemispheric speech circuitry [32, 41•]. Modified MIT protocols have been also been proposed and researched [36•] showing positive outcomes.

Recent research by Stahl et al. [43•] proposes that the element of rhythm may be equally or more important than the element of melody as originally has been thought. The hemispheric shift was originally proposed as a putative mechanism by the originators of MIT in the early 1970s. The mechanism for the functional re-routing of speech pathways was thought to be triggered mainly by the use of melody and singing as core musical elements engaging predominantly the right hemisphere. Recent work studies show, however, that the element of rhythm-using metronomic pacing, rhythmic hand tapping, and rhythmic speaking during MIT-may be equally or even more important to access right- hemispheric language resources.

The following elements in MIT may be considered the main mechanisms engaging preferentially right hemispheric networks for speech production:

- In melodic-rhythmic intonation vocal output is slower than when spoken. Singing is characterized by syllables being lengthened, chunked, and patterned, contributing to speed reduction in vocal output. The right hemisphere is better suited to process slowly modulating signals. Thus, translating spoken language into musical prosody prefentially activates right hemispheric language networks [49]

- Processing of music engages right hemispheric networks, thus, helping to bypass damaged left hemispheric language networks

- Rhythmic pacing and entrainment predominantly engages right hemispheric networks in auditory, prefrontal and parietal regions [65]

- Left hand tapping activates right hemispheric language networks since spoken language and arm gestures are controlled by the same motor control network [50]

\section{Studies in Cognitive Rehabilitation}

One of the most promising applications of music to cognitive training in stroke rehabilitation may be in the area of musical neglect training (MNT) which is one of the standardized techniques in NMT. MNT addresses hemispatial neglect and uses active performance exercises on musical instruments (tone bars or drums) that are structured in time, tempo, and rhythm, and are set up in appropriate spatial configurations to focus attention of the neglected or unattended visual field. A second application type in MNT consists of receptive music listening to stimulate hemispheric brain arousal while engaging in exercises addressing visual neglect or inattention [51-53]. Very interesting data were provided by Hommel [52] on the beneficial effect of musical stimulation for overcoming visual neglect as a result of right hemispheric lesions due to stroke or traumatic brain injury. Music stimuli were superior to other sensory and cognitive cues, such as instructions and speech or tactile cues. The researchers' rationale focused on the well-documented specific arousal effect of music on the right brain hemisphere that is lesioned in visual neglect states. This rationale would also be well supported by the theoretically established link between arousal and neglect in general, which has also been confirmed by research data. Robertson et al. [54] have shown that unilateral neglect can be dramatically altered by 

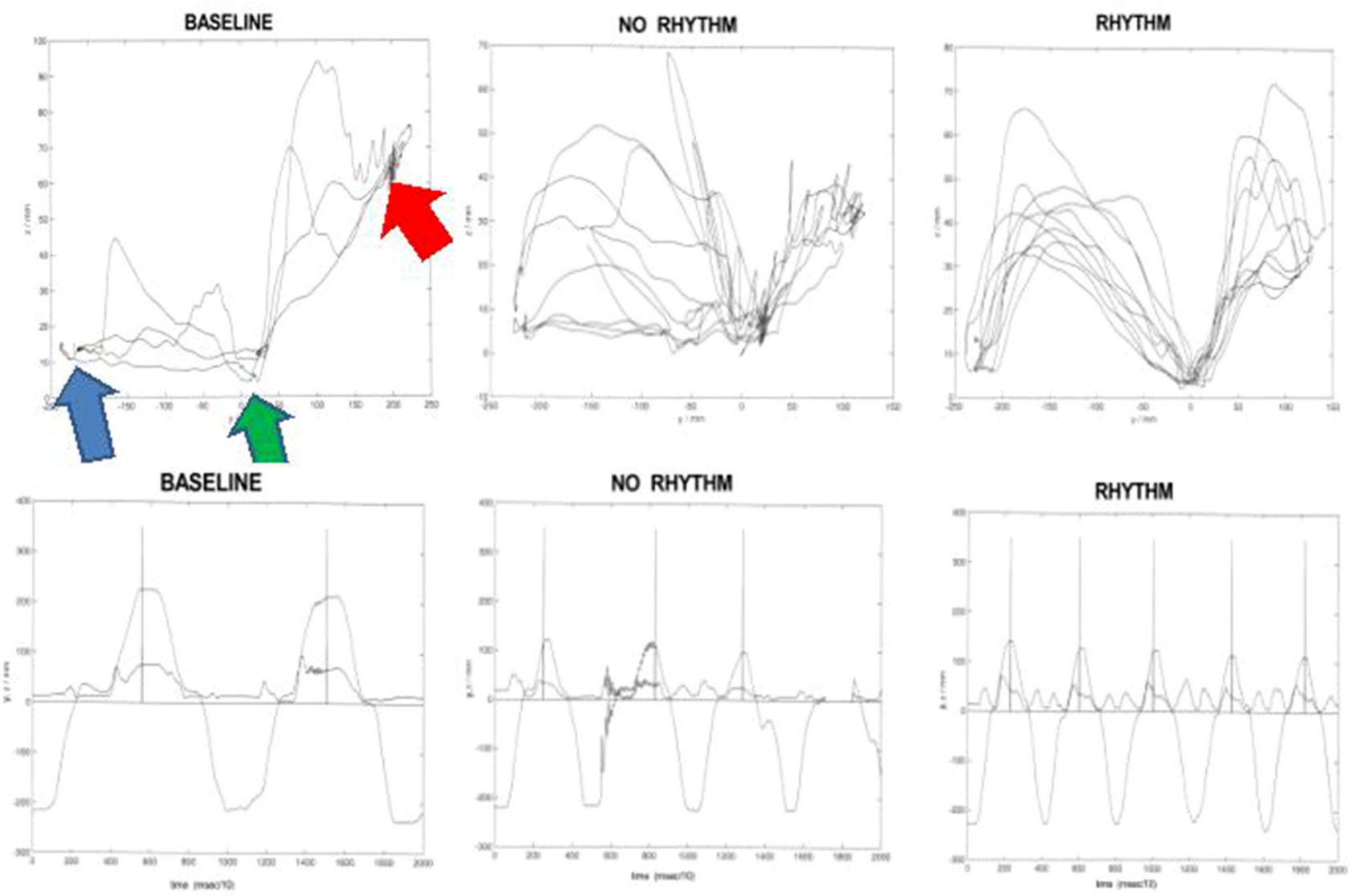

Fig. 1 Kinematic recording of hemiparetic arm reaching movement across three target circles on a table top, $5 \mathrm{~cm}$ circle diameter (blue arrow starting circle, green arrow second circle, red arrow third circle on ramp). Baseline movement without practice. No Rhythm after 3 min of uncued practice. Rhythm after 3 min of rhythmically cued practice (metronome frequency set at baseline frequency). Top row wrist marker movement on sagittal plane, bottom row y/z plane shown on $\mathrm{y}$-axis combined across time shown on $\mathrm{x}$-axis. Clearly visible is the more stable and normalized trajectory and reduction in trajectory variability across repetitions after the rhythmic practice (Color figure online)

tone were both presented in contralesional space. A research study in Japan by Noto et al. [59] reported that a patient with left spatial neglect improved attention toward the left side on a cancellation test after having trained playing a xylophone which was reversely placed with ascending keys from right to left. Using a similar training method, Kouya and Saito [60] reported that patients with left spatial neglect extended their personal hygiene activities to the left side of the face after training with a xylophone again set in reverse and by having the patients play melodies which emphasized ascending pitch patterns, thus, moving attention and arm movement from the right side to neglect left side (Fig. 1).

Abiru et al. [61] reported improvements in performance on a line cancellation test and flower drawing test as well as reductions in left side collision during wheelchair movement after exercises playing tone bars in spatial arrangements adjusted to the severity of patients' neglect. All these studies employ similar protocols with exercises involving 
instrumental playing in various spatial arrangements to emphasize the neglect side or arranging chord progressions or melodic patterns which move attention and movement progressively from the attended visual field into the neglect field.

\section{Summary}

Basic and clinical neuroscience research in music and brain function has driven a paradigmatic change in music therapy from a socio-cultural base, using interpretative models of music, to a neuroscience base using active perception and performance models. NMT has established evidence-based therapeutic techniques to re-train and re-educate brain and behavior functions in neurologic disorders and injuries, particularly in the area of motor recovery in stroke [62••, $63,64]$. Furthermore, neuroimaging studies have demonstrated a clearly-defined network of parietal, cerebellar, and frontal areas involved in the processing of rhythmicity [6567]. In this paper we have reviewed the research evidence for motor, speech/language, and cognitive intervention techniques in stroke rehabilitation. There is considerable evidence that NMT is an effective therapeutic technique in stroke rehabilitation.

\section{Compliance with Ethics Guidelines}

Conflict of Interest $\mathrm{MH}$ Thaut and GC McIntosh declare no conflict of interest.

Human and Animal Rights and Informed Consent This article does not contain any studies with human or animal subjects performed by any of the authors.

\section{References}

Papers of particular interest, published recently, have been highlighted as:

- Of importance

-• Of major importance

1. Paltsev YI, Elner AM. Change in the functional state of the segmental apparatus of the spinal cord under the influence of sound stimuli and its role in voluntary movement. Biophysics. 1967;12:1219-26.

2. Rossignol S, Melvill Jones G. Audiospinal influences in man studied by the H-reflex and its possible role in rhythmic movement synchronized to sound. Electroencephalogr Clin Neurophysiol. 1976;41:83-92.

3. Thaut MH, McIntosh GC, Prasas SG, Rice RR. The effect of rhythmic auditory cuing on stride and EMG patterns in normal gait. J Neurol Rehabil. 1992;6:185-90.
4. Thaut MH, McIntosh GC, Prassas SG, Rice RR. The effect of auditory rhythmic cuing on stride and EMG patterns in hemiparetic gait of stroke patients. J Neurol Rehabil. 1993;7:9-16.

5. Prassas S, Thaut M, McIntosh G, Rice R. Effect of auditory rhythmic cuing on gait kinematic parameters of stroke patients. Gait Posture. 1997;6:218-23.

6. Thaut MH, McIntosh GC, Rice RR. Rhythmic facilitation of gait training in hemiparetic stroke rehabilitation. J Neurol Sci. 1997; 151:207-12.

7. - Thaut MH, Leins AK, Rice RR, Argstatter H, Kenyon GP, McIntosh GC, Bolay V and Fetter M. Rhythmic auditory stimulation improves gait more than NDT/Bobath training in nearambulatory patients early post stroke: a single blind randomized trial. Neurorehabil Neural Repair. 2007; 21:455-459. A multicenter randomized control trial showing significantly higher improvements in gait over NDT/Bobath in hemiparetic stroke rehabilitation.

8. Schauer ML, Mauritz KH. Musical motor feedback (MMF) in walking of hemiparetic stroke patients: randomized trials of gait improvement. Clin Rehabil. 2003;17:713-22.

9. Schauer ML, Steingrueber W, Mauritz KH. Die Wirkung von Musik auf die Symmetrie des Gehens von Schlaganfallpatienten auf dem Laufband. Biomed Tech. 1996;41:291-6.

10. Ford M, Wagenaar R, Newell K. The effects of auditory rhythm and instruction on walking patterns in individuals post stroke. Gait Posture. 2007;26:150-5.

11. Abiru M, Nakano C, Kikuchi Y, Tokita K, Mihara Y, Fujimoto M, Mihara B. The effects of RAS in gait dusturbance caused by thalamic hemorrhage: a case study. Music Med. 2009;2:9-13.

12. Roerdink M, Lamoth CJC, Kwakkel G, van Wieringen PCW, Beek PJ. Gait coordination after stroke: benefits of acoustically paced treadmill walking. Phys Ther. 2007;87:1009-22.

13. Roerdink M, Lamoth CJC, van Kordelaar J, Elich P, Konijnenbelt M, Kwakkel G, Beek PJ. Rhythm perturbations in acoustically paced treadmill walking after stroke. Neurorehabil Neural Repair. 2009;23:668-78.

14. - Roerdink M. Walking to the beat of a different drum: practical implications for the use of acoustic rhythm in gait rehabilitation. Gait Posture.2011; 33:690-694. Practical consideration for functional gait training in neurorehabilitation using Rhythmic Auditory Stimulation.

15. Thaut MH, Miller RA, Schauer LM. Multiple synchronization strategies in rhythmic sensorimotor tasks: phase vs period correction. Biol Cybern. 1998;79:241-50.

16. Kenyon GP, Thaut MH. A measure of kinematic limb instability modulation by rhythmic auditory stimulation. J Biomech. 2000;33:1319-23.

17. Thaut MH, Kenyon GP, Hurt CP, McIntosh GC, Hoemberg V. Kinematic optimization of spatiotemporal patterns in paretic arm training with stroke patients. Neuropsychologia. 2002;40: 1073-81.

18. Malcolm MP, Massie C, Thaut MH. Rhythmic auditory motor entrainment improves hemiparetic arm kinematics during reaching movements. Top Stroke Rehabil. 2009;16:69-79.

19. - Senesac CR, Davis S and Richards L. Generalization of a modified rhythmic bilateral training in stroke. Hum Movem Sci. 2010; 26:137-148. Findings that improvements in during repetitive cyclical bilateral arm training cued rhythmically by metronome cues (Patterned Sensory Enhancement) transfer to functional improvements on gross motor tests.

20. Whitall J, McCombe WS, Silver KH, Macko RF. Repetitive bilateral arm training with rhythmic auditory cueing improves motor function in chronic hemiparetic stroke. Stroke. 2000;31:2390-5.

21. Luft AR, McCombe-Waller S, Whitall J, Forrester LW, Macko R, Sorkin JD, Schulz JB, Goldberg AP, Hanley DF. Repettitve 
bilateral arm training and motor cortex activation in chronic stroke: a randomized controlled trial. $\mathrm{J}$ Am Med Assoc. 2004;292:1853-61.

22. McCombe WS, Harris-Love M, Liu W, Whitall J. Temporal coordination of the arms during bilateral simultaneous and sequential movements in patients with chronic hemiparesis. Exp Brain Res. 2006;168:450-4.

23. Schneider S, Soenle PW, Altenmueller E, Muente TF. Using musical instruments to improve motoro skill recovery following stroke. J Neurol. 2007;254:1339-46.

24. Yoo J. The role of therapeutic instrumental music performance in hemiparetic arm rehabilitation. Music Ther Perspect. 2009; 27:16-24

25. Altenmueller E, Marco-Pallares J, Muente TF, Schneider S. Neural reorganization underlies improvement in stroke-induced motor dysfunction by music supported therapy. Ann N Y Acad Sci. 2010;1169:395-405.

26. - Schneider S. Music supported training is more efficient than functional motor training for recovery of fine motor skills in stroke patients. Music Percept. 2010; 27:713-722. Data supporting the advantage of therapeutic instrumental music playing for fine motor recovery post stroke over conventional physical therapy training.

27. Rojo N, Amenqual J, Juncadella M, Rubio F, Camara E, MarcoPallares J, Schneider S, Veciana M, Montero J, Mohammadi B, Altenmueller E, Grau C, Muente TF, Rodriguez-Fornells A. Music supported therapy induces plasticity in the sensorimotor cortex in chronic stroke: a single case study using multimodal imaging. Brain Inj. 2011;25:787-93.

28. • Grau-Sanchez J, Amenqual JL, Rojo N, Veciana M, Montero J, Rubio F, Altenmueller E, Muente TF and Rodriguez-Fornells A.Plasticity in the sensorimotor cortex induced by Music supported therapy in stroke patients: a TMS study. Front Hum Neurosci. 2013. doi:10.3389/fnhum2013.00494. Supportive evidence that functional irmprovements occur after Therapeutic Instrumental Playing after accompanied by neuroplastic changes in sensorimotor cortex.

29. Albert M, Sparks R, Helm N. Melodic intonation therapy for aphasics. Arch Neuro. 1973;29:130-1.

30. Sparks RW, Helm N, Albert M. Aphasia rehabilitation resulting from melodic intonation therapy. Cortex. 1974;10:313-6.

31. Sparks RW, Holland AL. Method: melodic intonation therapy for aphasia. J Speech Hear Disord. 1976;41:298-300.

32. Belin P, Van Eeckhout P, Zilbovicius M, Remy P, François C, Guillaume S, Chain F, Rancurel G, Sampson Y. Recovery from nonfluent aphasia after melodic intonation therapy. Neurology. 1996;47:1504-11.

33. Bonakdarpour B, Eftekharzadeh A, Ashayeri H. Melodic intonation therapy with Persian aphasic patients. Aphasiology. 2003; 17:75-95.

34. Boucher V, Garcia JL, Fleurant J, Paradis. Variable efficacy of rhythm and tone in melody-based intervetions: implications of the assumption of a right-hemisphere facilitation in non-fluent aphasia. Aphasiology. 2001;15:131-49.

35. - Breier JL, Randle S, maher LM \& Papanicolaou AC. Changes in maps of language activity activation following melodic intonation therapy using magnetoencephalography: two case studies. J Clin Exp Neuropsychol. 2010;32:309-314. Findings supporting the reactivation of left hemispheric language areas in successful speech recovery induced by melodic intonation therapy.

36. • Conklyn D, Novak E, Boissy A, Bethoux F \& Chemali K. The effects of modified melodic intonation therapy on non-fluent aphasia: a pilot study. J Speech Lang Hear Res. 2012;55:1463-1471. Demonstrates successful speech recovery for a larger clinical sample using modified melodic intonation therapy.
37. Goldfarb R, Bader E. Espousing melodic intonation therapy in aphasia rehabilitation: a case study. Int $\mathrm{J}$ Rehabil Res. 1979;2:333-42.

38. Hebert S, Racette A, Gagnon L, Peretz I. Revisiting the dissociation between singing and speaking in expressive aphasia. J Neurol. 2003;126:1838-51.

39. Popovici M. Melodic intonation therapy in the verbal decoding of aphasics. Rev Roum Neurol Psychiatr. 1995;33:57-97.

40. Racette A, Bard C, Peretz I. Making non-fluet aphasics speak: sing along. Brain. 2006;129:2571-84.

41. - Schlaug G, Marchina S \& Norton A. Evidence for plasticity in white-matter tracts of patients with chronic Broca's aphasia undergoing intense intonation-based speech therapy. Ann New York Acad Sci. 2009;1169:385-394. Findings demonstrating neuroplasticity induced by intensive melodic intonation therapy training.

42. Seki K, Sugishita M. Japanese-applied melodic intonation therapy for Broca's aphasia. Brain Nerve (No to Skinkei). 1983;35:1031-7.

43. - Stahl B, Kotz SA Henseler I, Turner R \& Geyer S. Rhythm in disguise: why singing may not hold the key to recovery from aphasia. Brain. 2011;134:3083-3093. Data supporting a critical role for rhythm in speech recovery after melodic intonation therapy.

44. Straube T, Schulz A, Geipel K, Mentzel HJ, Miltner WH. Dissociation between singing and speaking in expressive aphasia: the role of song familiarity. Neuropsychologia. 2008;46:1505-12.

45. Wilson SJ. Preserved singing in aphasia: a case study of the efficacy of melodic intonation therapy. Music Percept. 2006;24:23-6.

46. Yamadori A, Osumi Y, Masuhara S, Okubo M. Preservation of singing in Broca's aphasia. J Neurol Neurosurg Psychiatr. 1977;40:221-4.

47. Yamaguchi S, Akanuma K, Hatayama Y, Otero M, Meguro K. Singing therapy can be effective for a patient with severe nonfluent aphasia. Int J Rehabil Res. 2012;35:78-81.

48. Stewart L, Walsh V, Frith U, Rothwell J. Transcranial magnetic stimulation produces speech arrest but not song arrest. Ann N Y Acad Sci. 2001;930:433-5.

49. Patel AD. The relationship of music to the melody of speech and to syntactic processing disorders in aphasia. Ann N Y Acad Sci. 2005;1060:59-70.

50. Gentilucci M, Dalla Volta R. Spoken language and arm gestures are controlled by the same motor control system. Q J Exp Psychol. 2008;61:944-57.

51. Frassinetti F, Bolognini N, Ladavos E. Enhancement of visual perception by crossmodal visual-auditory interaction. Exp Brain Res. 2002;147:332-43.

52. Hommel M, Peres B, Pollak P, Memin B. Effects of passive tactile and auditory stimuli on left visual neglect. Arch Neurol. 1990;47:573-6.

53. Thaut MH. Rhythm, music, and the brain. New York: Taylor \& Francis; 2005

54. Robertson IH, Mattingley JM, Rorden C, Driver J. Phasic alerting of neglect patients overcomes their spatial deficit in visual awareness. Nature. 1998;395:169-72.

55. Robertson IH, Nico D, Hood B. The intention to act improves unilateral neglect: two demonstrations. NeuroReport. 1995;17:246-8.

56. Frassinetti F, Pavani F, Ladavos E. Acoustical vision of neglected stimuli: interaction among spatially convergent audio-visual inputs in neglect patients. J Cognit Neurosci. 2002;14:62-9.

57. • Soto D, Funes MJ, Guzman-Garcia A, Warbick T, Rohhstein P \& Humphreys GW. Pleasant music overcomes the loss of awareness in patients with visual neglect. Proc Nat Acad Sci. 2012;106:6011-6016. The study finds support for music stimulation to overcome hemispatial neglect, proposing an additional 
role for recruitment of attention resources through affective responses to preferred music.

58. Van Vleet TM, Robertson LC. Cross-modal interactions in time and space: auditory influence on visual attention in hemispatial neglect. J Cogn Neurosci. 2006;18:1368-79.

59. Noto S, Mouri F, Amimoto K, Sugimoto S, Futaki T. Effect of "xylophone therapy" for a patient of unilateral spatial neglect. J Jpn Occup Ther Assoc. 1999;18:126-33.

60. Kouya I, Saito Y. A report with regard to the efficacy of the Japanese drum therapy carried out for the rehabilitation of a cerebral apoplexy patient. Jpn J Music Ther. 2004;4:198-207.

61. Abiru M, Mihara Y, Kikuchi. The effects of Neurologic music therapy on hemispatial neglect in a hemiparetic stroke patient. A case study. Neurol Med. 2007;67:88-94.

62. •- Hoemberg V. Neurorehabilitation approaches to facilitate motor recovery. In: Barnes MP and Good DC, editors. Handbook of neurology, vol 110. New York: Elsevier; 2013. pp. 161-174. Current review on state of the art therapies for motor recovery.
Includes NMT techniques for hemiparetic gait and arm rehabilitation.

63. Hummelsheim H. Rationales for improving motor function. Curr Opin Neurol. 1999;12:697-701.

64. Jeffrey DR, Good DC. Rehabilitation of the stroke patient. Curr Opin Neurol. 1995;8:62-8.

65. Stephan KM, Thaut MH, Wunderlich G, Schicks W, Tian B, Tellmann L, Schmitz T, Herzog H, McINtosh GC, Seitz RJ, Hoemberg V. Conscious and subconscious sensorimotor synchronization: prefrontal cortex and the inf;uence of awareness. Neuroimage. 2002;15:345-52.

66. Thaut MH. Neural basis of rhythmic timing networks in the human brain. Ann N Y Acad Sci. 2003;999:364-73.

67. Thaut MH, Stephan KM. Wunderlich, Schicks W, Tellmann L, Herzog, H, McIntosh GC, Seitz RJ, Hoemberg V. Distinct cortico-cerebellar activations in rhythmic auditory motor synchronization. Cortex. 2008;45:44-53. 